\title{
An Approximation to Zeros of the Riemann Zeta Function Using Fractional Calculus
}

\author{
A. Torres-Hernandez ${ }^{1, *}$, F. Brambila-Paz ${ }^{2}$ \\ ${ }^{1}$ Department of Physics, Faculty of Science - UNAM, Mexico \\ ${ }^{2}$ Department of Mathematics, Faculty of Science - UNAM, Mexico
}

Received February 5, 2021; Revised April 7, 2021; Accepted April 18, 2021

Cite This Paper in the following Citation Styles

(a): [1] A. Torres-Hernandez, F. Brambila-Paz, "An Approximation to Zeros of the Riemann Zeta Function Using Fractional Calculus," Mathematics and Statis-tics, Vol.9, No.3, pp. 309-318, 2021. DOI: 10.13189/ms.2021.090312.

(b): A. Torres-Hernandez, F. Brambila-Paz, (2021). An Approximation to Zeros of the Riemann Zeta Function Using Fractional Calculus. Mathematics and Statistics, 9(3), 309-318. DOI: 10.13189/ms.2021.090312.

Copyright $@ 2021$ by authors, all rights reserved. Authors agree that this article remains permanently open access under the terms of the Creative Commons Attribution License 4.0 International License

\begin{abstract}
In this paper an approximation to the zeros of the Riemann zeta function has been obtained for the first time using a fractional iterative method which originates from a unique feature of the fractional calculus. This iterative method, valid for one and several variables, uses the property that the fractional derivative of constants are not always zero. This allows us to construct a fractional iterative method to find the zeros of functions in which it is possible to avoid expressions that involve hypergeometric functions, Mittag-Leffler functions or infinite series. Furthermore, we can find multiple zeros of a function using a singe initial condition. This partially solves the intrinsic problem of iterative methods, which in general is necessary to provide $N$ initial conditions to find $N$ solutions. Consequently the method is suitable for approximating nontrivial zeros of the Riemann zeta function when the absolute value of its imaginary part tends to infinity. Some examples of its implementation are presented, and finally 53 different values near to the zeros of the Riemann zeta function are shown.
\end{abstract}

Keywords Fractional Derivative, Fractional Iterative Method, Riemann Zeta Function

\section{Introduction}

A classical problem in mathematics which is of common interest in physics and engineering is finding the set of zeros of a function $f: \Omega \subset \mathbb{R}^{n} \rightarrow \mathbb{R}^{n}$, that is,

$$
\operatorname{ker}(f):=\{\xi \in \Omega:\|f(\xi)\|=0\},
$$

where $\|\cdot\|: \mathbb{R}^{n} \rightarrow \mathbb{R}$ denotes any vector norm. Although finding the zeros of a function may seem like a simple problem, many times it involves solving an algebraic equation system given by the following expression

$$
\left\{\begin{array}{c}
{[f]_{1}(x)=0} \\
{[f]_{2}(x)=0} \\
\vdots \\
{[f]_{n}(x)=0}
\end{array}\right.
$$

where $[f]_{k}: \mathbb{R}^{n} \rightarrow \mathbb{R}$ denotes the $k$-th component of the function $f$. It should be mentioned that system (2) may represent a linear system or a nonlinear system. The function that has the set of zeros of most interest in mathematics, being possibly the most famous set of zeros, corresponds to the Riemann zeta function 


$$
\zeta(x)=\sum_{k=1}^{\infty} \frac{1}{k^{x}} .
$$

The Riemann hypothesis, under the assumption that it is true, is responsible for giving a general expression to the zeros of the function $\zeta$, and may be written compactly as

$$
\text { If } \xi \in \mathbb{C} \Rightarrow \xi \in \operatorname{ker}(\zeta) \Leftrightarrow \xi=\left\{\begin{array}{cc}
-2 x, & \text { for all } x \in \mathbb{N} \\
0.5+x i, & \text { for some } x \in \mathbb{R}
\end{array},\right.
$$

when $\xi \neq-2 x$ it is known as a nontrivial zero of the function $\zeta$. In general, it is necessary to use numerical methods of the iterative type to construct a sequence $\left\{x_{i}\right\}_{i=0}^{\infty}$, that under certain conditions, allows to approximate to values $\xi \in \operatorname{ker}(\zeta)$ with $\xi \neq-2 x$, that is,

$$
x_{i} \underset{i \rightarrow \infty}{\longrightarrow} \xi \in \operatorname{ker}(\zeta) .
$$

If we want to find $N$ values $\xi \in \operatorname{ker}(\zeta)$, it is necessary to give $N$ initial conditions $x_{0}$. This is an intrinsic problem of iterative methods, because time must first be spent determining to initial condition $x_{0}$ before beginning to search the values $\xi$. Furthermore, it is sometimes necessary that the initial conditions are near to the searched values to guarantee convergence, that is,

$$
\left|x_{0}-\xi\right|<\epsilon \Rightarrow x_{i} \underset{i \rightarrow \infty}{\longrightarrow} \xi \in \operatorname{ker}(\zeta) .
$$

These problems are partially solved using fractional iterative methods, because they can determine $N$ values $\xi$ using a single initial condition $x_{0}$, and the initial condition does not need to be near the searched values. In this paper a fractional iterative method is used, which has the peculiarity that it uses the fractional derivative of constants to find the zeros of functions, and due to this peculiarity when implementing the fractional derivative operator it is possible to avoid expressions involving hypergeometric functions, Mittag-Leffler functions or infinite series. Then, it is an ideal iterative method to work with functions that can be expressed in terms of a series, as is the case of the Riemann zeta function, or to solve nonlinear systems in several variables.

\section{Fixed Point Method}

Let $\Phi: \mathbb{R}^{n} \rightarrow \mathbb{R}^{n}$ be a function. It is possible to build a sequence $\left\{x_{i}\right\}_{i=0}^{\infty}$ defining the following iterative method

$$
x_{i+1}:=\Phi\left(x_{i}\right),
$$

if it is true that the sequence $\left\{x_{i}\right\}_{i=0}^{\infty}$ converges to a value $\xi \in \mathbb{R}^{n}$, and if the functions $\Phi$ is continuous around $\xi$, it holds that

$$
\xi=\lim _{i \rightarrow \infty} x_{i+1}=\lim _{i \rightarrow \infty} \Phi\left(x_{i}\right)=\Phi\left(\lim _{i \rightarrow \infty} x_{i}\right)=\Phi(\xi),
$$

the above result is the reason by which the method (5) is known as fixed point method. Furthermore, the function $\Phi$ is called an iteration function. The following theorem allows characterizing the order of convergence of an iteration function $\Phi$ with its derivatives [1, 2, 3, 4]. Before continuing, we need to consider the following multi-index notation. Let $\mathbb{N}_{0}$ be the set $\mathbb{N} \cup\{0\}$, if $\gamma \in \mathbb{N}_{0}^{n}$, then

$$
\left\{\begin{array}{c}
\gamma !:=\prod_{k=1}^{n}[\gamma]_{k} !, \quad|\gamma|:=\sum_{k=1}^{n}[\gamma]_{k}, \quad x^{\gamma}:=\prod_{k=1}^{n}[x]_{k}^{[\gamma]_{k}} \\
\frac{\partial^{\gamma}}{\partial x^{\gamma}}:=\frac{\partial^{|\gamma|}}{\partial[x]_{1}^{[\gamma]_{1}} \partial[x]_{2}^{[\gamma]_{2}} \cdots \partial[x]_{n}^{[\gamma]_{n}}}
\end{array}\right.
$$


Theorem 2.1. Let $\Phi: \Omega \subset \mathbb{R}^{n} \rightarrow \mathbb{R}^{n}$ be an iteration function with a fixed point $\xi \in \Omega$. Assuming that $\Phi$ is $p$-times differentiable in $\xi$ for some $p \in \mathbb{N}$, and furthermore

$$
\left\{\begin{array}{cc}
\frac{\partial^{\gamma}[\Phi]_{k}(\xi)}{\partial x^{\gamma}}=0, \forall k \geq 1 \text { and } \forall|\gamma|<p, & \text { if } p \geq 2 \\
\left\|\Phi^{(1)}(\xi)\right\|<1, & \text { if } p=1
\end{array}\right.
$$

where $\Phi^{(1)}$ denotes the Jacobian matrix of the function $\Phi$, then $\Phi$ is (locally) convergent of (at least) order $p$.

Proof. The proof may be found in the reference [4].

The next corollary follows from the previous theorem.

Corollary 2.2. Let $\Phi: \mathbb{R}^{n} \rightarrow \mathbb{R}^{n}$ be an iteration function. If $\Phi$ defines a sequence $\left\{x_{i}\right\}_{i=0}^{\infty}$ such that $x_{i} \rightarrow \xi$, and if the following condition is fulfilled

$$
\lim _{x \rightarrow \xi}\left\|\Phi^{(1)}(x)\right\| \neq 0
$$

then $\Phi$ has an order of convergence (at least) linear in $B(\xi ; \delta)$.

\section{Riemann-Liouville Fractional Operators}

One of the fundamental operators of fractional calculus is the operator Riemann-Liouville fractional integral, which is defined as follows [5, 6]

$$
{ }_{a} I_{x}^{\alpha} f(x):=\frac{1}{\Gamma(\alpha)} \int_{a}^{x}(x-t)^{\alpha-1} f(t) d t,
$$
[5. 7]

which is a fundamental piece to build the operator Riemann-Liouville fractional derivative, which is defined as follows

$$
{ }_{a} D_{x}^{\alpha} f(x):=\left\{\begin{array}{cc}
{ }_{a} I_{x}^{-\alpha} f(x), & \text { if } \alpha<0 \\
\frac{d^{n}}{d x^{n}}\left({ }_{a} I_{x}^{n-\alpha} f(x)\right), & \text { if } \alpha \geq 0
\end{array},\right.
$$

where $n=\lceil\alpha\rceil$ and ${ }_{a} I_{x}^{0} f(x):=f(x)$. Applying the operator 111 with $a=0$ to the function $x^{\mu}$, with $\mu>-1$, we obtain the following result [4]:

$$
{ }_{0} D_{x}^{\alpha} x^{\mu}=\frac{\Gamma(\mu+1)}{\Gamma(\mu-\alpha+1)} x^{\mu-\alpha}, \quad \alpha \in \mathbb{R} \backslash \mathbb{Z} .
$$

\section{Fractional Pseudo-Newton Method}

Although the interest in fractional calculus has mainly focused on the study and development of techniques to solve differential equation systems of order non-integer [5, 6, 7, 8, 9, 10, 11]. Over the years, iterative methods have also been developed that use the properties of fractional derivatives to solve algebraic equation systems [4, 12, 13, 14, 15, 16, 17, 18, 19, 20]. These methods may be called fractional iterative methods, which under certain conditions, may accelerate their speed of convergence with the implementation of the Aitken's method [2, 4], recently these methods have been useful in the search for solutions to algebraic equation systems related to hybrid solar receivers [3, 19]. It should be noted that depending on the definition of fractional derivative used, fractional iterative methods have the particularity that they may be used of local form [12] or of global form [18]. Furthermore, for some definitions of fractional derivative, it is fulfilled that the derivative of the order $\alpha$ of a constant is different from zero, that is, 


$$
\partial_{k}^{\alpha} c:=\frac{\partial^{\alpha}}{\partial[x]_{k}^{\alpha}} c \neq 0, \quad c=\text { constant }
$$

where $\partial_{k}^{\alpha}$ denotes any fractional derivative applied only in the component $k$, that does not cancel the constants (for example: Riesz, Grünwald-Letnikov, Riemann-Liouville, etc. [5, 6, 7, 8, 9, 10, 11]), and that fulfills the following continuity relation with respect to the order $\alpha$ of the derivative

$$
\lim _{\alpha \rightarrow 1} \partial_{k}^{\alpha} c=\partial_{k} c .
$$

Considering a function $\Phi:(\mathbb{R} \backslash \mathbb{Z}) \times \mathbb{C}^{n} \rightarrow \mathbb{C}^{n}$, recently a fractional iterative method has been proposed that has already been implemented in an engineering application, which is called the fractional pseudo-Newton method and is defined by the following expression [3]:

$$
x_{i+1}:=\Phi\left(\alpha, x_{i}\right)=x_{i}-P_{\epsilon, \beta}\left(x_{i}\right) f\left(x_{i}\right), \quad i=0,1,2 \cdots,
$$

where $P_{\epsilon, \beta}\left(x_{i}\right)$ is a matrix evaluated in the value $x_{i}$, which is given by the following expression

$$
P_{\epsilon, \beta}\left(x_{i}\right):=\left(\left[P_{\epsilon, \beta}\right]_{j k}\left(x_{i}\right)\right)=\left(\partial_{k}^{\beta\left(\alpha,\left[x_{i}\right]_{k}\right)} \delta_{j k}+\epsilon \delta_{j k}\right)_{x_{i}},
$$

where

$$
\partial_{k}^{\beta\left(\alpha,\left[x_{i}\right]_{k}\right)} \delta_{j k}:=\frac{\partial^{\beta\left(\alpha,\left[x_{i}\right]_{k}\right)}}{\partial[x]_{k}^{\beta\left(\alpha,\left[x_{i}\right]_{k}\right)}} \delta_{j k}, \quad 1 \leq j, k \leq n,
$$

with $\delta_{j k}$ the Kronecker delta, $\epsilon$ a positive constant $\ll 1$, and $\beta\left(\alpha,\left[x_{i}\right]_{k}\right)$ a function defined as follows

$$
\beta\left(\alpha,\left[x_{i}\right]_{k}\right):=\left\{\begin{array}{lll}
\alpha, & \text { if } & \left|\left[x_{i}\right]_{k}\right| \neq 0 \\
1, & \text { if } & \left|\left[x_{i}\right]_{k}\right|=0
\end{array} .\right.
$$

Due to the part of the integral operator that fractional derivatives usually have, we consider in the matrix (16) that each fractional derivative is obtained for a real variable $[x]_{k}$, and if the result allows it, this variable is subsequently substituted by a complex variable $\left[x_{i}\right]_{k}$, that is,

$$
P_{\epsilon, \beta}\left(x_{i}\right):=\left.P_{\epsilon, \beta}(x)\right|_{x \longrightarrow x_{i}}, \quad x \in \mathbb{R}^{n}, \quad x_{i} \in \mathbb{C}^{n} .
$$

It should be mentioned that the value $\alpha=1$ in (18), is taken to avoid the discontinuity that is generated when using the fractional derivative of constants in the value $x=0$. Furthermore, since in the previous method $\left\|\Phi^{(1)}(\alpha, \xi)\right\| \neq 0$ if $\|f(\xi)\|=0$, from the Corollary 2.2. any sequence $\left\{x_{i}\right\}_{i=0}^{\infty}$ generated by the iterative method (15) has an order of convergence (at least) linear.

\subsection{Some Examples}

Instructions for implementing the method $(15)$ along with information to provide values $\alpha \in[-2,2] \backslash \mathbb{Z}$ are found in the reference [18]. On the other hand, it should be mentioned that the method 15 may be implemented through recursive programming in a way analogous to that presented in the reference [21]. For rounding reasons, only for the examples the following function is defined

$$
\operatorname{Rnd}_{m}\left([x]_{k}\right):=\left\{\begin{array}{cl}
\operatorname{Re}\left([x]_{k}\right), & \text { if }\left|\operatorname{Im}\left([x]_{k}\right)\right| \leq 10^{-m} \\
{[x]_{k},} & \text { if }\left|\operatorname{Im}\left([x]_{k}\right)\right|>10^{-m}
\end{array} .\right.
$$

Combining the function 20 , with the method $[15$, the following iterative method is defined

$$
x_{i+1}:=\operatorname{Rnd}_{5}\left(\Phi\left(\alpha, x_{i}\right)\right), \quad i=0,1,2 \cdots .
$$


Example 4.1. Let $\left\{f_{k}\right\}_{k=0}^{\infty}$ be a sequence of functions, with

$$
f_{k}(x)=-\gamma-\log (x)-\sum_{m=1}^{k} \frac{(-1)^{m} x^{2 m}}{2 m \Gamma(2 m+1)} \underset{k \rightarrow \infty}{\longrightarrow} \int_{x}^{\infty} \frac{\cos (t)}{t} d t
$$

where $\gamma$ is the Euler-Mascheroni constant [22]. Then considering the value $k=50$, the initial condition $x_{0}=0.018$ is chosen to use the iterative method given by (21) along with fractional derivative given by (12). Consequently, we obtain the results of the Table 1

\begin{tabular}{c|ccccc}
\hline & $\alpha$ & $x_{n}$ & $\left\|x_{n}-x_{n-1}\right\|_{2}$ & $\left\|f_{50}\left(x_{n}\right)\right\|_{2}$ & $n$ \\
\hline 1 & -1.11419 & 15.7703495 & $6.60000 e-7$ & $9.53989 e-9$ & 33 \\
2 & -1.10571 & 22.03613996 & $3.20000 e-7$ & $3.67622 e-9$ & 29 \\
3 & -1.09148 & 3.38418043 & $3.00000 e-7$ & $2.13662 e-9$ & 23 \\
4 & -1.09147 & 9.52557552 & $4.40000 e-7$ & $6.51952 e-9$ & 23 \\
5 & -1.00855 & 28.30949579 & $1.10000 e-7$ & $8.74154 e-7$ & 110 \\
6 & 0.24869 & $-18.65007933+4.7866359 i$ & $5.12250 e-7$ & $9.98818 e-7$ & 61 \\
7 & 0.25206 & $-24.9715585+5.07028087 i$ & $4.21900 e-7$ & $8.51586 e-7$ & 61 \\
8 & 0.25269 & $-12.29980885+4.3899063 i$ & $5.19230 e-7$ & $9.35313 e-7$ & 61 \\
9 & 0.25298 & $-18.65007948-4.78663644 i$ & $4.41022 e-7$ & $8.66794 e-7$ & 64 \\
10 & 0.28661 & $-5.86092713+3.72436638 i$ & $5.74282 e-7$ & $9.63373 e-7$ & 57 \\
11 & 0.29031 & $-24.97155803-5.07028097 i$ & $3.26497 e-7$ & $7.97508 e-7$ & 64 \\
12 & 0.30109 & $-12.29980928-4.38990586 i$ & $4.44072 e-7$ & $9.93021 e-7$ & 66 \\
13 & 0.31605 & $-5.86092745-3.72436648 i$ & $4.79270 e-7$ & $8.82199 e-7$ & 62 \\
14 & 1.11281 & 31.44800139 & $2.00000 e-8$ & $1.56267 e-7$ & 3 \\
15 & 1.14602 & 0.61650487 & $2.60000 e-7$ & $8.14734 e-7$ & 33 \\
\hline
\end{tabular}

Table 1. Results obtained using the iterative method 21 with $\epsilon=e-3$.

Example 4.2. Let $\left\{f_{k}\right\}_{k=0}^{\infty}$ be a sequence of functions, with

$$
f_{k}(x)=\frac{\pi}{2}-\sum_{m=0}^{k} \frac{(-1)^{m} x^{2 m+1}}{(2 m+1) \Gamma(2 m+2)} \underset{k \rightarrow \infty}{\longrightarrow} \int_{x}^{\infty} \frac{\sin (t)}{t} d t .
$$

Then considering the value $k=50$, the initial condition $x_{0}=1.85$ is chosen to use the iterative method given by (21) along with fractional derivative given by (12). Consequently, we obtain the results of the Table 2

\begin{tabular}{c|ccccc}
\hline & $\alpha$ & $x_{n}$ & $\left\|x_{n}-x_{n-1}\right\|_{2}$ & $\left\|f_{50}\left(x_{n}\right)\right\|_{2}$ & $n$ \\
\hline 1 & -0.83718 & 23.60399266 & $4.10000 e-7$ & $9.80551 e-9$ & 30 \\
2 & -0.81526 & 29.87824476 & $7.30000 e-7$ & $8.19133 e-7$ & 273 \\
3 & -0.71339 & 17.33566366 & $4.10000 e-7$ & $2.48591 e-8$ & 34 \\
4 & -0.71324 & 11.08303768 & $3.70000 e-7$ & $2.67499 e-8$ & 29 \\
5 & -0.71174 & 4.89383571 & $5.10000 e-7$ & $4.87621 e-8$ & 24 \\
6 & 0.36251 & $-12.29964074-4.38965942 i$ & $4.41814 e-7$ & $9.42697 e-7$ & 42 \\
7 & 0.36333 & $-31.27978791-5.29112884 i$ & $4.31045 e-7$ & $5.52647 e-7$ & 391 \\
8 & 0.36684 & $-24.97153098-5.07020771 i$ & $3.56931 e-7$ & $7.71425 e-7$ & 42 \\
9 & 0.36976 & $-18.65001989-4.78651292 i$ & $3.80132 e-7$ & $8.88913 e-7$ & 40 \\
10 & 0.38451 & $-24.97153097+5.07020788 i$ & $2.05913 e-7$ & $5.36982 e-7$ & 44 \\
11 & 0.38646 & $-18.65002028+4.78651268 i$ & $2.30000 e-7$ & $5.54454 e-7$ & 42 \\
12 & 0.40682 & $-12.29964106+4.38965969 i$ & $4.49110 e-7$ & $9.97466 e-7$ & 37 \\
13 & 0.44711 & $-5.86005858-3.72373544 i$ & $4.72017 e-7$ & $9.90371 e-7$ & 41 \\
14 & 0.48437 & $-5.86005854+3.72373556 i$ & $4.52217 e-7$ & $9.82000 e-7$ & 38 \\
15 & 0.55885 & $-31.27978639+5.2911368 i$ & $5.50000 e-7$ & $3.69789 e-7$ & 183 \\
16 & 1.41172 & 1.92644561 & $1.10000 e-7$ & $9.97696 e-7$ & 196 \\
\hline
\end{tabular}

Table 2. Results obtained using the iterative method 21 with $\epsilon=e-3$. 
Example 4.3. Let $f$ be a function, with

$$
f(x)=\left(\begin{array}{c}
\frac{1}{2}[x]_{1}\left(\sin \left([x]_{1}[x]_{2}\right)-1\right)-\frac{1}{4 \pi}[x]_{2} \\
\left(1-\frac{1}{4 \pi}\right)\left(e^{2[x]_{1}}-e\right)+e\left(\frac{1}{\pi}[x]_{2}-2[x]_{1}\right)
\end{array}\right) .
$$

Then the initial condition $x_{0}=(0.86,0.86)^{T}$ is chosen to use the iterative method given by 21) along with fractional derivative given by (12). Consequently, we obtain the results of the Table 3

\begin{tabular}{c|cccccc}
\hline & $\alpha$ & {$\left[x_{n}\right]_{1}$} & {$\left[x_{n}\right]_{2}$} & $\left\|x_{n}-x_{n-1}\right\|_{2}$ & $\left\|f\left(x_{n}\right)\right\|_{2}$ & $n$ \\
\hline 1 & 0.69508 & $1.01828092+0.52158397 i$ & $5.18478971-3.76689418 i$ & $1.15758 e-7$ & $7.24108 e-7$ & 48 \\
2 & 0.69632 & $-0.13780201+0.87180277 i$ & $2.16460973+4.68221216 i$ & $1.31909 e-7$ & $9.55148 e-7$ & 80 \\
3 & 0.7283 & $-0.13780202-0.87180273 i$ & $2.16460988-4.68221226 i$ & $9.11043 e-8$ & $8.81449 e-7$ & 100 \\
4 & 0.72889 & -0.15442216 & 1.14021866 & $6.22977 e-7$ & $8.30511 e-7$ & 60 \\
5 & 0.75757 & $1.01828092-0.5215839 i$ & $5.18479004+3.76689413 i$ & $9.21954 e-8$ & $9.83408 e-7$ & 80 \\
6 & 0.78188 & $-0.20477864-1.30850366 i$ & $2.21623485-7.86783099 i$ & $5.56776 e-8$ & $9.92736 e-7$ & 246 \\
7 & 0.82863 & $1.14584377-0.68994257 i$ & $8.09450013+5.9960712 i$ & $3.31662 e-8$ & $9.77549 e-7$ & 193 \\
8 & 0.86097 & $1.14584377+0.68994256 i$ & $8.09450017-5.99607116 i$ & $2.64575 e-8$ & $9.42041 e-7$ & 249 \\
9 & 1.11159 & 1.70987637 & -18.87534307 & $1.41421 e-8$ & $9.92487 e-7$ & 447 \\
10 & 1.14766 & 1.48216448 & -8.41311536 & $1.41421 e-8$ & $8.86632 e-7$ & 233 \\
11 & 1.17262 & $-1.36674692+0.07786741 i$ & $-5.76423+0.47853094 i$ & $2.00000 e-8$ & $9.92337 e-7$ & 394 \\
12 & 1.18538 & $-1.36674698-0.07786726 i$ & $-5.76422966-0.4785315 i$ & $2.23607 e-8$ & $9.88600 e-7$ & 387 \\
13 & 1.19954 & 1.57643706 & -12.098725 & $1.41421 e-8$ & $7.09538 e-7$ & 386 \\
14 & 1.20058 & 1.64946521 & -15.55495398 & $1.41421 e-8$ & $9.10544 e-7$ & 465 \\
15 & 1.2852 & $-0.76073057+0.14192444 i$ & $-2.11123992+0.82667655 i$ & $1.02470 e-7$ & $8.39720 e-7$ & 97 \\
16 & 1.28668 & $-0.76073047-0.14192446 i$ & $-2.11123884-0.8266763 i$ & $2.74044 e-7$ & $7.74152 e-7$ & 81 \\
17 & 1.29642 & 1.34362303 & -4.29400761 & $7.61577 e-8$ & $4.60872 e-7$ & 92 \\
\hline
\end{tabular}

Table 3. Results obtained using the iterative method 21] with $\epsilon=e-3$.

\section{Approximation to zeros of the Riemann zeta function}

A systematic study of the Riemann zeta function is beyond the intent of this paper. However, the basic information necessary to approximate their zeros using the iterative method (21) will be presented. Let $\zeta: \Omega \subset \mathbb{C} \backslash\{1\} \rightarrow \mathbb{C}$ be the Riemann zeta function with $\Omega=\{x \in \mathbb{C}: \operatorname{Re}(x)>1\}$. The function $\zeta$ is defined as follows

$$
\zeta(x)=\sum_{k=1}^{\infty} \frac{1}{k^{x}}
$$

the previous expression may be extended for all $x \in \mathbb{C} \backslash\{1\}$ by analytic continuation. With which it is possible to obtain the following functional equation

$$
\zeta(x)=2^{x} \pi^{x-1} \sin \left(\frac{\pi x}{2}\right) \Gamma(1-x) \zeta(1-x) .
$$

On the other hand, there exists a series version of the Riemann zeta function, which has the characteristic of being globally convergent for all $x \in \mathbb{C} \backslash\{1\}$. This version of the function $\zeta$ was conjectured by Konrad Knopp and proved by Helmut Hasse in 1930 [23], and it is given by the following expression

$$
\zeta(x)=\frac{1}{1-2^{1-x}} \sum_{m=0}^{\infty} \frac{1}{2^{m+1}} \sum_{p=0}^{m}(-1)^{p}\left(\begin{array}{c}
m \\
p
\end{array}\right)(p+1)^{-x} .
$$

It is necessary to mention that the expression 24] is very useful, because it allows us to make numerical approximations of the nontrivial zeros of the Riemann zeta function.

Example 5.1. Let $\left\{f_{k}\right\}_{k=0}^{\infty}$ be a sequence of functions, with 


$$
f_{k}(x)=\frac{1}{1-2^{1-x}} \sum_{m=0}^{k} \frac{1}{2^{m+1}} \sum_{p=0}^{m}(-1)^{p}\left(\begin{array}{c}
m \\
p
\end{array}\right)(p+1)^{-x} \underset{k \rightarrow \infty}{\longrightarrow} \zeta(x) .
$$

Then considering the value $k=50$, the initial condition $x_{0}=0.5+31.51 i$ is chosen to use the iterative method given by (21) along with fractional derivative given by (12). Consequently, we obtain the results of the Table 4

\begin{tabular}{|c|c|c|c|c|c|}
\hline & $\alpha$ & $x_{n}$ & $\left\|x_{n}-x_{n-1}\right\|_{2}$ & $\left\|f_{50}\left(x_{n}\right)\right\|_{2}$ & $n$ \\
\hline 1 & -1.13348 & -5.99997526 & $9.90000 e-7$ & $1.46057 e-7$ & 266 \\
\hline 2 & -0.94547 & -2.00001275 & $8.00000 e-7$ & $3.88212 e-7$ & 146 \\
\hline 3 & -0.88567 & -9.9999519 & $7.90000 e-7$ & $5.72079 e-7$ & 54 \\
\hline 4 & -0.15505 & $0.49999963+14.13472531 i$ & $9.89798 e-7$ & $3.22385 e-7$ & 22 \\
\hline 5 & -0.06835 & $0.54743578+947.15979693 i$ & $8.59593 e-7$ & $3.79999 e-7$ & 349 \\
\hline 6 & -0.06585 & $0.48286572-305.3825058 i$ & $8.95377 e-7$ & $4.74515 e-7$ & 251 \\
\hline 7 & -0.06135 & $0.47107264-430.95892398 i$ & $9.80051 e-7$ & $4.94661 e-7$ & 108 \\
\hline 8 & -0.02125 & $0.5460791+321.6875341 i$ & $7.92023 e-7$ & $5.38613 e-7$ & 61 \\
\hline 9 & -0.01965 & $0.48286548+305.38250571 i$ & $6.32456 e-7$ & $1.92115 e-7$ & 49 \\
\hline 10 & -0.01685 & $0.61664333+276.09845269 i$ & $5.95483 e-7$ & $2.99694 e-7$ & 99 \\
\hline 11 & -0.01275 & $0.58077376+224.41293459 i$ & $9.49210 e-7$ & $8.04311 e-7$ & 267 \\
\hline 12 & -0.01015 & $0.56816592+192.65292746 i$ & $9.39628 e-7$ & $7.02867 e-7$ & 192 \\
\hline 13 & -0.00755 & $0.46159425+158.41373653 i$ & $8.31505 e-7$ & $4.66903 e-7$ & 175 \\
\hline 14 & -0.00545 & $0.56284646+133.97913455 i$ & $9.39840 e-7$ & $7.30271 e-7$ & 213 \\
\hline 15 & -0.00465 & $0.48007233+123.75232554 i$ & $9.48472 e-7$ & $8.25385 e-7$ & 278 \\
\hline 16 & -0.00195 & $0.4913738+95.32674434 i$ & $5.73847 e-7$ & $1.78959 e-7$ & 172 \\
\hline 17 & 0.01795 & $0.50002627-37.5861995 i$ & $9.40213 e-7$ & $9.17598 e-7$ & 255 \\
\hline 18 & 0.02145 & $0.49137435-95.32674404 i$ & $9.88130 e-7$ & $3.70864 e-7$ & 125 \\
\hline 19 & 0.02165 & $0.50000116-32.93506385 i$ & $8.62670 e-7$ & $6.14360 e-7$ & 120 \\
\hline 20 & 0.03195 & $0.49999981-25.01085752 i$ & $7.35391 e-7$ & $3.11793 e-7$ & 53 \\
\hline 21 & 0.03295 & $0.46159416-158.41373657 i$ & $8.10555 e-7$ & $3.57361 e-7$ & 139 \\
\hline 22 & 0.03305 & $0.50000005-30.42487677 i$ & $9.08020 e-7$ & $6.57518 e-7$ & 73 \\
\hline 23 & 0.03425 & $0.50007248-40.91868687 i$ & $6.35059 e-7$ & $3.03925 e-7$ & 87 \\
\hline 24 & 0.03785 & $0.50000027-21.02203963 i$ & $9.83107 e-7$ & $3.08350 e-7$ & 31 \\
\hline 25 & 0.04495 & $0.50000036-14.13472527 i$ & $8.24197 e-7$ & $3.03119 e-7$ & 21 \\
\hline 26 & 0.06185 & $0.50000011+21.02203962 i$ & $3.21403 e-7$ & $1.28471 e-7$ & 40 \\
\hline 27 & 0.06735 & $0.40419552+1185.59789987 i$ & $5.52268 e-7$ & $4.10670 e-7$ & 67 \\
\hline 28 & 0.14095 & $0.50689898-2878.59861379 i$ & $3.55106 e-7$ & $9.12771 e-7$ & 330 \\
\hline 29 & 0.24136 & $0.44802022-495.84458602 i$ & $2.32594 e-7$ & $8.59529 e-7$ & 60 \\
\hline 30 & 0.24176 & $0.54285436-466.79078671 i$ & $2.08806 e-7$ & $9.24091 e-7$ & 218 \\
\hline 31 & 0.24256 & $0.58122569-404.58829656 i$ & $2.62488 e-7$ & $9.89996 e-7$ & 354 \\
\hline 32 & 0.24306 & $0.59895653-373.61214166 i$ & $2.26716 e-7$ & $9.90256 e-7$ & 464 \\
\hline 33 & 0.24346 & $0.46960335-346.68847181 i$ & $2.10950 e-7$ & $8.74078 e-7$ & 496 \\
\hline 34 & 0.24766 & $0.46263812-146.98942449 i$ & $3.08869 e-7$ & $8.33117 e-7$ & 496 \\
\hline 35 & 0.24806 & $0.56284605-133.97913398 i$ & $3.61248 e-7$ & $9.02931 e-7$ & 478 \\
\hline 36 & 0.24836 & $0.48007222-123.75232428 i$ & $2.72029 e-7$ & $7.75292 e-7$ & 484 \\
\hline 37 & 0.25106 & $0.50076291-56.43890631 i$ & $3.64005 e-7$ & $8.55540 e-7$ & 354 \\
\hline 38 & 0.25156 & $0.50021423-43.32696237 i$ & $4.86004 e-7$ & $7.09618 e-7$ & 312 \\
\hline 39 & 0.26886 & $0.50000042+25.01085761 i$ & $3.53553 e-7$ & $5.54795 e-7$ & 94 \\
\hline 40 & 0.28486 & $0.50007238+40.91868722 i$ & $3.13847 e-7$ & $8.22684 e-7$ & 51 \\
\hline 41 & 0.29196 & $0.50002634+37.5861994 i$ & $3.74433 e-7$ & $9.61617 e-7$ & 46 \\
\hline 42 & 0.29836 & $0.50021436+43.32696223 i$ & $2.56125 e-7$ & $5.52253 e-7$ & 42 \\
\hline 43 & 0.30096 & $0.50125709+49.77289402 i$ & $2.41661 e-7$ & $6.80998 e-7$ & 63 \\
\hline 44 & 0.30126 & $0.49834763+52.96633019 i$ & $2.45153 e-7$ & $4.93711 e-7$ & 43 \\
\hline 45 & 0.30136 & $0.5007636+56.43890622 i$ & $2.72029 e-7$ & $9.06750 e-7$ & 69 \\
\hline 46 & 0.30196 & $0.49914035+60.81054836 i$ & $2.87924 e-7$ & $9.28287 e-7$ & 66 \\
\hline 47 & 0.30686 & $0.49914001-60.81054769 i$ & $2.86531 e-7$ & $9.54106 e-7$ & 59 \\
\hline 48 & 0.30696 & $0.47366839-67.04250707 i$ & $2.62488 e-7$ & $8.53872 e-7$ & 94 \\
\hline 49 & 0.30776 & $0.49834757-52.96632988 i$ & $2.64764 e-7$ & $5.86654 e-7$ & 288 \\
\hline 50 & 0.30786 & $0.50125707-49.77289365 i$ & $2.34094 e-7$ & $6.76399 e-7$ & 325 \\
\hline 51 & 0.31156 & $0.47366808+67.04250713 i$ & $2.78927 e-7$ & $9.54910 e-7$ & 44 \\
\hline 52 & 0.32336 & $0.50000099+30.42487725 i$ & $2.59422 e-7$ & $9.16200 e-7$ & 120 \\
\hline 53 & 0.32386 & $0.50000112+32.93506408 i$ & $2.73130 e-7$ & $7.59689 e-7$ & 40 \\
\hline
\end{tabular}

Table 4. Results obtained using the iterative method 21 with $\epsilon=e-3$.

The comments below are made under the assumption that the Riemann hypothesis given in equation (3) is true. Table 4 shows certain $x_{n}$ values with $\left|\operatorname{Re}\left(x_{n}\right)-0.5\right| \geq 0.1$, this is partly a consequence of the approximation of the function $\zeta$ by means of the function $f_{k}$, because in general the following condition is true 


$$
\operatorname{ker}\left(f_{k}\right) \neq \operatorname{ker}(\zeta) \Leftrightarrow k<\infty .
$$

In addition, it is necessary to mention that the use of iterative methods does not guarantee that we can get as near as we want to the value $\xi$, normally it is only possible to determine a value $x_{n}$ near to the value $\xi$ given by the following expression

$$
x_{n}=\xi+\delta_{\xi}, \quad \delta_{\xi}=\delta_{\xi}(n), \quad\left\|\delta_{\xi}\right\|<1,
$$

considering 25], it is necessary to give a definition that allows us to characterize the behavior of a function with respect to the values $x_{n}$ in $\bar{B}\left(\xi ; \delta_{\xi}\right)$.

Definition 5.2. Let $f: \Omega \subset \mathbb{R}^{n} \rightarrow \mathbb{R}^{n}$ be a function with a value $\xi \in \Omega$ such that $\|f(\xi)\|=0$. If when doing $\xi \rightarrow \xi+\delta_{\xi}$, with $\left\|\delta_{\xi}\right\|<1$, it holds that

$$
\left\|f\left(\xi+\delta_{\xi}\right)\right\|=\left\|\delta_{f}\right\|<1,
$$

then the function $f$ is (locally) stable with respect to the value $\xi$ in $\bar{B}\left(\xi, \delta_{\xi}\right)$.

The condition (26), implies that for a function $f$ to be (locally) stable, it is necessary that a slight perturbation $\delta_{\xi}$ in its zeros does not generate a great perturbation $\delta_{f}$ in its images. To try to observe the stability of function $\zeta$, we may consider $\delta=10^{-12}$ and the following values

$$
\begin{aligned}
& x_{n_{1}}=-40-1 \delta \Rightarrow\left|\zeta\left(x_{n_{1}}\right)\right| \approx 4.8 \times 10^{3} \quad\left|x_{n_{1}}=-60-1 \delta \Rightarrow\right| \zeta\left(x_{n_{1}}\right) \mid \approx 5.3 \times 10^{21} \\
& x_{n_{2}}=-40+0 \delta \Rightarrow \quad\left|\zeta\left(x_{n_{2}}\right)\right|=0 \quad x_{n_{2}}=-60+0 \delta \quad \Rightarrow \quad\left|\zeta\left(x_{n_{2}}\right)\right|=0 \quad . \\
& x_{n_{3}}=-40+1 \delta \Rightarrow\left|\zeta\left(x_{n_{3}}\right)\right| \approx 4.8 \times 10^{3} \quad x_{n_{3}}=-60+1 \delta \Rightarrow\left|\zeta\left(x_{n_{3}}\right)\right| \approx 5.3 \times 10^{21}
\end{aligned}
$$

By performing multiple examples, it is possible to show for the trivial zeros of function $\zeta$ the validity of the following affirmation:

$$
\text { If } \xi=-2 x \text { with } x \in \mathbb{N} \Rightarrow \zeta \text { is (locally) unstable in } \xi \text { if } x \rightarrow \infty .
$$

The previous affirmation implies that it is complicated to make numerical approximations to trivial zeros of $\zeta$ by iterative methods using an initial condition $x_{0} \neq-2 x$ with $x \in \mathbb{N}$ when $x \rightarrow \infty$. Furthermore, since the behavior of the nontrivial zeros of function $\zeta$ is not completely determined, which is one of the many reasons for which the Riemann hypothesis remains an unsolved problem [23, 24, 25], it is possible to generate the following question:

$$
\text { If } \xi=0.5+x i \text { with } x \in \mathbb{R} \Rightarrow \text { Is } \zeta \text { (locally) unstable in } \xi \text { if }|x| \rightarrow \infty \text { ? }
$$

\section{Conclusions}

Due to the fact that the derivatives of constant functions are identically zero functions in conventional calculus, it is difficult to imagine them as tools that may be used to approximate the zeros of more complicated functions. However, in the fractional calculus, the derivatives of constant functions may be used to approximate the zeros of more complicated functions and even nondifferentiables. It is necessary to mention that constant functions are the easiest functions to work with in fractional calculus, since the fractional derivatives of more complicated functions are expressed in many occasions in terms of Mittag-Leffler functions, hypergeometric functions or infinite series. In this paper, an approximation to the zeros of the Riemann zeta function has been obtained for the first time using fractional derivatives of constant functions.

Since the fractional derivative of constants are not always zero. Allows us to construct a fractional iterative method to find the zeros of functions in which it is possible to avoid expressions that involve hypergeometric functions, Mittag-Leffler functions or infinite series. Then, it is an ideal iterative method to work with functions that can be expressed in terms of a series, as is the case of the Riemann zeta function. The method does not explicitly depend on the fractional derivative of the function for which zeros are sought, then it is an ideal iterative method for solving nonlinear systems in several variables.

The fractional iterative methods are efficient at finding multiple zeros of a function using a single initial condition, in addition to having the particularity of finding complex zeros of polynomials using real initial conditions. For this reason, they are iterative methods suitable for working with functions that have a large number of zeros, as is the case of the Riemann zeta function. 


\section{REFERENCES}

[1] Robert Plato. Concise numerical mathematics, pages 105-120. Number 57. American Mathematical Soc., 2003.

[2] Josef Stoer and Roland Bulirsch. Introduction to numerical analysis, volume 12, pages 289-348. Springer Science \& Business Media, 2013.

[3] A. Torres-Hernandez, F. Brambila-Paz, P. M. Rodrigo, and E. De-la-Vega. Reduction of a nonlinear system and its numerical solution using a fractional iterative method. Journal of Mathematics and Statistical Science, 6:285-299, 2020. http: //www.ss-pub.org/journals/jmss/vol-6/vol-6-issue-10-october-2020/.

[4] A. Torres-Hernandez, F. Brambila-Paz, U. Iturrarán-Viveros, and R. Caballero-Cruz. Fractional newton-raphson method accelerated with aitken's method. Axioms, 10(2):1-25, 2021. DOI: 10.3390/axioms10020047.

[5] Rudolf Hilfer. Applications of fractional calculus in physics, pages 3-73. World Scientific, 2000.

[6] Keith Oldham and Jerome Spanier. The fractional calculus theory and applications of differentiation and integration to arbitrary order, volume 111, pages 25-121. Elsevier, 1974.

[7] A.A. Kilbas, H.M. Srivastava, and J.J. Trujillo. Theory and Applications of Fractional Differential Equations, pages 69132. Elsevier, 2006.

[8] Kenneth S. Miller and Bertram Ross. An introduction to the fractional calculus and fractional differential equations, pages 1-125. Wiley-Interscience, 1993.

[9] Fernando Brambila. Fractal Analysis: Applications in Physics, Engineering and Technology, pages 4-42. IntechOpen, 2017.

[10] Carlos Alberto Torres Martínez and Carlos Fuentes. Applications of radial basis function schemes to fractional partial differential equations. Fractal Analysis: Applications in Physics, Engineering and Technology, pages 4-20, 2017. DOI: $10.5772 / 67892$.

[11] Benito F. Martínez-Salgado, Rolando Rosas-Sampayo, Anthony Torres-Hernández, and Carlos Fuentes. Application of fractional calculus to oil industry. Fractal Analysis: Applications in Physics, Engineering and Technology, pages 21-42, 2017. DOI: 10.5772/intechopen.68571

[12] Feng Gao, Xiaojun Yang, and Zongxin Kang. Local fractional newton's method derived from modified local fractional calculus. In 2009 International Joint Conference on Computational Sciences and Optimization, volume 1, pages 228-232. IEEE, 2009. DOI: 10.1109/CSO.2009.330.

[13] A. Torres-Hernandez and F. Brambila-Paz. Fractional newton-raphson method. Applied Mathematics and Sciences: An International Journal (MathSJ), 8:1-13, 2021. DOI: 10.5121/mathsj.2021.8101.

[14] Krzysztof Gdawiec, Wiesław Kotarski, and Agnieszka Lisowska. Visual analysis of the newton's method with fractional order derivatives. Symmetry, 11(9):1143, 2019. DOI: 10.3390/sym11091143.

[15] Krzysztof Gdawiec, Wiesław Kotarski, and Agnieszka Lisowska. Newton's method with fractional derivatives and various iteration processes via visual analysis. Numerical Algorithms, pages 1-58, 2020. DOI: 10.1007/s11075-020-00919-4.

[16] Ali Akgül, Alicia Cordero, and Juan R Torregrosa. A fractional newton method with 2 $\alpha$ th-order of convergence and its stability. Applied Mathematics Letters, 98:344-351, 2019. DOI: 10.1016/j.aml.2019.06.028.

[17] Alicia Cordero, Ivan Girona, and Juan R Torregrosa. A variant of chebyshev's method with $3 \alpha$ th-order of convergence by using fractional derivatives. Symmetry, 11(8):1017, 2019. DOI: 10.3390/sym11081017.

[18] A. Torres-Hernandez, F. Brambila-Paz, and E. De-la-Vega. Fractional newton-raphson method and some variants for the solution of nonlinear systems. Applied Mathematics and Sciences: An International Journal (MathSJ), 7:13-27, 2020. DOI: $10.5121 /$ mathsj.2020.7102.

[19] A. Torres-Hernandez, F. Brambila-Paz, P. M. Rodrigo, and E. De-la-Vega. Fractional pseudo-newton method and its use in the solution of a nonlinear system that allows the construction of a hybrid solar receiver. Applied Mathematics and Sciences: An International Journal (MathSJ), 7:1-12, 2020. DOI: 10.5121/mathsj.2020.7201. 
[20] A. Torres-Hernandez, F. Brambila-Paz, and J. J. Brambila. A nonlinear system related to investment under uncertainty solved using the fractional pseudo-newton method. Journal of Mathematical Sciences: Advances and Application, 63:4153, 2020. http://scientificadvances.co.in/artical/1/285

[21] A. Torres-Hernandez. Code of multidimensional newton-raphson method using recursive programming, 2021. DOI: 10.13140/RG.2.2.12871.42407.

[22] G. Arfken and H.J. Weber. Mathematical Methods for Physicists, pages 560-565. 1985.

[23] David Joyner and Jon-Lark Kim. Selected unsolved problems in coding theory, pages 71-121. Springer Science \& Business Media, 2011.

[24] Xiao-Jun Yang. Non-trivial zeros of riemann's zeta function via revised euler-maclaurin-siegel and abel-plana summation formulas. 2018. arXiv preprint arXiv:1811.02418

[25] Xiao-Jun Yang. The nontrivial zeros of completed zeta function and riemann hypothesis. scientiairanica, 26:2167-2175, 2019. http://scientiairanica.sharif.edu/article_21465.html.

[26] Muhammad Asim Khan and Norhashidah $\mathrm{Hj}$. Mohd Ali. Fourth-order compact iterative scheme for the two-dimensional time fractional sub-diffusion equations. Mathematics and Statistics, 8(2A):52-57, 2020. DOI: 10.13189/ms.2020.081309.

[27] Shams A Ahmed and Mohamed Elbadri. Solution of newell-whitehead-segal equation of fractional order by using sumudu decomposition method. Mathematics and Statistics, 8(6):631-636, 2020. DOI: 10.13189/ms.2020.080602. 